ARTICLE

\title{
Empowering alcohols as carbonyl surrogates for Grignard-type reactions
}

Chen-Chen Li (10 ${ }^{1}$, Haining Wang ${ }^{1}$, Malcolm M. Sim ${ }^{1}$ Zihang Qiu (D) ${ }^{1}$, Zhang-Pei Chen ${ }^{1}$, Rustam Z. Khaliullin ${ }^{1} \&$ Chao-Jun Li (iD ${ }^{1 凶}$

The Grignard reaction is a fundamental tool for constructing C-C bonds. Although it is widely used in synthetic chemistry, it is normally applied in early stage functionalizations owing to poor functional group tolerance and less availability of carbonyls at late stages of molecular modifications. Herein, we report a Grignard-type reaction with alcohols as carbonyl surrogates by using a ruthenium(II) PNP-pincer complex as catalyst. This transformation proceeds via a carbonyl intermediate generated in situ from the dehydrogenation of alcohols, which is followed by a Grignard-type reaction with a hydrazone carbanion to form a C-C bond. The reaction conditions are mild and can tolerate a broad range of substrates. Moreover, no oxidant is involved during the entire transformation, with only $\mathrm{H}_{2}$ and $\mathrm{N}_{2}$ being generated as byproducts. This reaction opens up a new avenue for Grignard-type reactions by enabling the use of naturally abundant alcohols as starting materials without the need for pre-synthesizing carbonyls.

\footnotetext{
${ }^{1}$ Department of Chemistry and FQRNT Centre for Green Chemistry and Catalysis, McGill University, 801 Sherbrooke Street West, Montreal, QC H3A OB8, Canada. ${ }_{\text {email: cj.li@mcgill.ca }}$
} 
$\mathrm{n}$ tandem with the significant advancements of biological and pharmaceutical technologies, the role of organic chemists has evolved beyond the discovery of new chemical transformations. Developments such as rapid and direct late-stage functionalizations of large molecules have shown great potentials, with increased significance of organic reactions ${ }^{1}$. The Grignard reaction is a fundamental transformation in chemical synthesis and has been continuously developed over the past century. Its importance is attributed to the reaction's versatility and capacity to form $\mathrm{C}-\mathrm{C}$ bonds, leading to the formation of secondary and tertiary alcohols ${ }^{2-7}$. A key limitation of this reaction, however, is its instability and broad reactivity. In addition, classical synthetic methods used to transform carbonyl compounds often requires the participation of oxidants, many of which are hazardous and have poor functional group tolerance ${ }^{7}$. Insofar, the Grignard reaction has typically been limited to early-stage construction instead of the direct late-stage modification of complex molecules or natural products.

In contrast, alcohols are among the most naturally abundant functional groups, which are commonly found in biomass and natural products. The direct transformation of alcohols into $\mathrm{C}-\mathrm{C}$ bonds has been a long pursuit of synthetic chemists ${ }^{8-11}$. This type of transformation would be an especially vital tool for the latestage functionalization of alcohol-containing natural products and pharmaceuticals. Furthermore, this type of transformation will contribute greatly to the future sustainability of chemical syntheses by minimizing the number of steps required (Fig. 1). Motivated by these potential benefits, we contemplated the possibility of using alcohols as surrogates of aldehydes and ketones for the Grignard-type reaction via the in situ formal "dehydrogenation" of alcohol catalyzed by transition metals ${ }^{8}$. Early extensive studies have shown that ruthenium(II) and other transition-metal complexes are efficient catalysts for the aerobic oxidation of alcohols to carbonyls ${ }^{12-16}$, which indicates the potential for hydroxyl groups to act as carbonyl surrogates. This strategy, however, has been limited to the hydrogen-borrowing aldol reactions, Michael additions ${ }^{17-22}$ and reductive aminations $^{23}$. The use of alcohols as carbonyl surrogates for a Grignardtype reaction has never been successfully demonstrated. In order to successfully develop this reaction, two key challenges must be overcome: (1) the incompatibility of both the acidic alcohol proton and the oxidant with Grignard reagents ${ }^{2}$, and (2) the possible transmetallation of organometallic reagents with ruthenium catalysts which in turn attenuates the activity of catalyst ${ }^{24}$.

Hydrazones are known as halogen-free, easily accessible and traceless carbanion equivalents. Their use as "carbanions" in various reactivity has been developed by our group over the past several years ${ }^{25-28}$. Our early studies have shown that hydrazones react efficiently with carbonyls via a 1,2-addition catalyzed by ruthenium(II)-phosphine complexes, and such a reactivity can even be successfully applied in synergistic relay reactions ${ }^{29}$. More importantly, unlike the classical Grignard reagents, hydrazones show unique tolerance towards acidic protons such as hydroxyl and amino groups. Herein, we report a unique Grignard-type reaction with alcohol as a carbonyl surrogate and hydrazones as carbanion equivalents using a ruthenium(II) catalyst (Fig. 2b).

\section{Results}

Exploration of the hydride acceptor-system. A key step of this surrogate strategy was the in situ catalytic generation of carbonyls from alcohols through their "dehydrogenation". Based on previous studies from our group ${ }^{30}$ and others ${ }^{31}, \beta$-hydride elimination of alcohols could be efficiently catalyzed by $\mathrm{Ru}(\mathrm{II})$ complexes with or without a stoichiometric amount of oxidants (hydride acceptors). The former has often been conducted under milder conditions, while the latter usually requires high temperatures with the use of special catalysts ${ }^{32-35}$. Thus, our investigation started by seeking a proper hydride acceptor. On the basis of our previous work ${ }^{27,29}$, a ruthenium(II)-bidentate phosphine system was first explored by varying the type of oxidants (hydride acceptors) used (Table 1). We observed a 9\% yield of the desired product in the absence of a hydride acceptor (Table 1, entry 1). Under such conditions, however, most of the alcohols remained unchanged while the hydrazone substrate had mostly undergone the competing Wolff-Kishner reduction (WK reduction), which led to an overall low efficiency for the Grignard-type $\mathrm{C}-\mathrm{C}$ bond formation. Inspired by the aerobic oxidation of alcohols, we then tested the reaction under air and $\mathrm{O}_{2}$ atmospheres, respectively; however, even lower yields were obtained in both cases (Table 1, entries 2 and 3). In the cases of NCS, isoprene and silver oxide as oxidants, no generation of the desired product was observed (Table 1, entries 4-6). These results indicated that either the ruthenium(II) catalyst or the hydrazone substrate was incompatible with strong oxidants. For this reason, weaker oxidants such as copper oxide and DCB (2,3-dichlorobutane) were then tested. As expected, the product yield showed a notable increase. The conversion efficiencies of the reactants, however, were still relatively low (Table 1 , entries 7 and 8 ). Nevertheless, these results suggested that weak oxidants can be tolerated in this $\mathrm{Ru}(\mathrm{II})$-bisphosphine system, albeit not significantly promoting the oxidation.

Exploration of oxidant-free and acceptorless system. The results above led us to consider an oxidant-free strategy in order to increase the efficiency of alcohol dehydrogenation, which will in turn allow the subsequent 1,2-addition of hydrazone by modifying the catalytic system. Upon carefully analyzing the results with the $\mathrm{Ru}(\mathrm{II})$-dcypf system, we attributed the main reason for the low efficiency to the inefficient kinetics of the dehydrogenation process. The extensive studies on Noyori-type reactions have

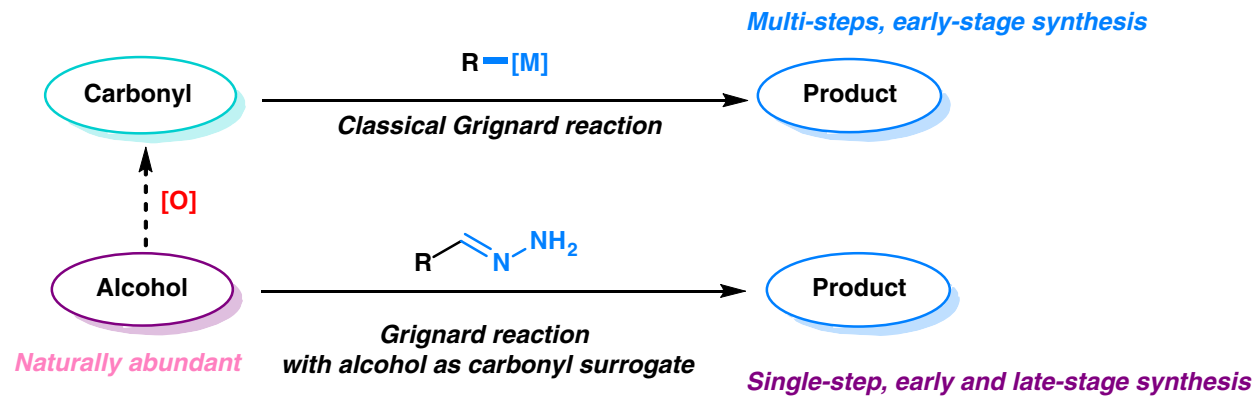

Fig. 1 Classical Grignard reaction and Grignard reaction with alcohol as carbonyl surrogate. This figure shows the comparison between classical Grignard reaction and the Grignard reaction with alcohol as carbonyl surrogate. It clearly shows that the latter is both step and atom economical. More importantly, using alcohol as carbonyl surrogate has a broad potential for late-stage functionalizations in synthetic chemistry. 

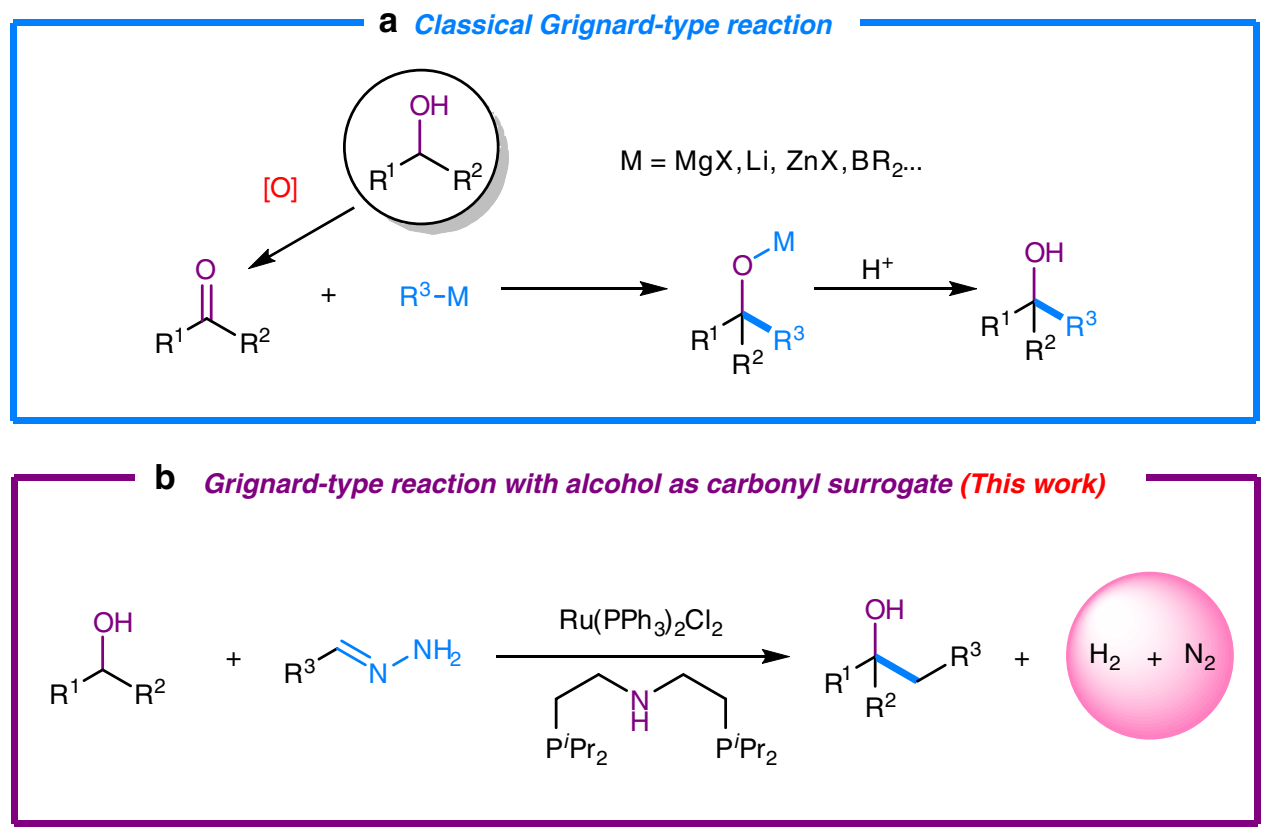

Fig. 2 Grignard-type reaction with alcohol as carbonyl surrogate. a Classical Grignard-type reaction uses carbonyls as starting materials which come from the oxidation of alcohols. Organometallic reagents, in this case, serve as carbanion equivalent. b Alcohol-surrogated Grignard-type reaction, reported in this article, directly uses alcohol as starting material with hydrazones as carbanion equivalent, which skips the process of oxidation and generates hydrogen gas and nitrogen gas as only byproducts.

shown that a mixture of phosphine and amine ligands could accelerate the hydrogenation and dehydrogenation processes due to the favored six-membered pericyclic transition state $^{36}$. Furthermore, most reported reactions concerning acceptorless dehydrogenation of alcohols require $\mathrm{P}-\mathrm{N}$ type ligands ${ }^{32-35}$. These studies inspired us to investigate alternative catalytic systems other than $\mathrm{Ru}(\mathrm{II})$-bisphosphine.

We started by using a well-defined Noyori-type ruthenium complex, $\mathrm{Ru}(\mathrm{dppf})(\mathrm{en}) \mathrm{Cl}_{2}$ (dppf = diphenylphosphinoethane; en = ethylenediamine). In addition, we increased the hydrazone substrate equivalence to 3.5 in order to minimize the Wolff-Kishner reduction of the hydrazone. This initial attempt, however, only increased the yield slightly (Table 2 , entry (1). We reasoned that since $\mathrm{Ru}(\mathrm{dppf})(\mathrm{en}) \mathrm{Cl}_{2}$ is a stable complex with a limited number of empty coordination sites, it cannot drive the whole cascade process to proceed efficiently. To solve this problem, PNP-pincer type ligands ${ }^{19,37-39}$ were then considered because: (1) with a tridentate structure, they can form more stable complexes with the metal center in order to stabilize the intermediates in the catalytic cycle, and (2) by occupying only three coordination sites, more space could be provided for the substrates and intermediates in order to facilitate the catalytic process. To our delight, the use of PNP-pincer type ligands indeed significantly increased the reactivity, among which bis[(2diisopropylphosphino]ethyl)amine (L3) provided the best result (Table 2, entry 4).

Although the yield was increased, there were still some notable side products being generated when using $\mathbf{L} \mathbf{3}$ as a ligand. One of the side products was the olefination product (3aa-s1) and the other was the hydrogen-borrowing hydrazination product (3aa-s2). Additionally, the Wolff-Kishner reduction also consumed all the remaining hydrazone before the complete conversion of the alcohol. We therefore carried out further optimizations in order to reduce the generation of these side products (Table 2, entries 5-9). The results showed that, by diluting the solution to $0.5 \mathrm{~mL}$, the side reactions (i.e., the WK reduction and hydrogenborrowing hydrazination) were significantly reduced without impeding the reactivity of the desired reaction. Furthermore, the dilution also enabled the alcohol to be fully consumed in the presence of a smaller amount of hydrazone. Under the optimized conditions, different pincer ligands such as PNN-type and $\mathrm{PN}_{\text {pyridine }} \mathrm{P}$-type ligands were investigated; however, none of them gave better results compared to L3 (Table 2, entries 10-12). Furthermore, pre-synthesizing the ruthenium complex with L3 gave almost the same results as the one generated in situ (Table 1, entry 13). In addition, we also tested a less bulky PNP ligand, bis [(2-diethylphosphino]ethyl)amine (L-Et), and produced a welldefined complex (Ru-PNP-2) to run the reaction (Table 2, entry 14). The result with this complex was similar to the Ru-L3 system. Later substrate scope studies also showed that both catalytic systems worked efficiently for primary alcohols. Thus, due to the fact that L-Et was less available, $\mathbf{L 3}$ was used as the optimized ligand in most of our later studies. The use of a less bulky pincer ligand, however, did increase the yield for bulkier secondary alcohols, which will be discussed later.

Investigation of the substrate scope. With the optimized reaction conditions in hand, the substrate scope investigation (Fig. 3) was started for the alcohol partners in which simple alcohols were tested first. The results showed that the linear aliphatic alcohols examined all underwent the reaction smoothly. Notably, longer aliphatic chains led to lower yields (3aa-3ac); however, the overall yields were generally high. Aliphatic alcohols substituted with methylthio (3ae) and -NBoc (3ah) were also compatible with this process and provided moderate to high yields of the desired products. Similar results were obtained for heterocyclic substituted alcohols (3ad-3ai). In order to investigate the potential application of this reaction for pharmaceutical or agricultural industries, we demonstrated that the fluorine-containing alcohol also underwent the Grignard-type reaction and provided a moderate yield (3hj). A noteworthy finding was that small molecular alcohols such as ethanol could also participate in this $\mathrm{C}-\mathrm{C}$ bond formation process at an elevated temperature (3hk). 


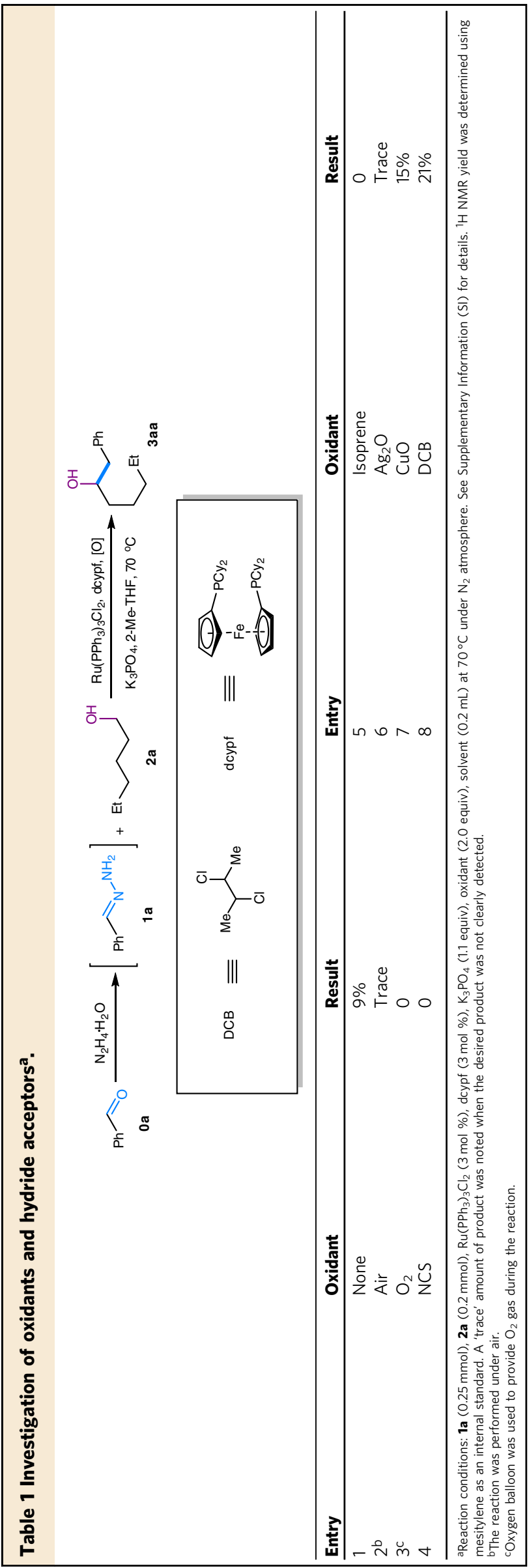

Benzyl alcohol and its derivatives were also effective substrates for this reaction; however, they generated more olefination products (3am-3ao). Increased steric effects suppressed this reaction as shown by the use of $\alpha$-substituted alcohols (3ap-3ar) with the exception of $\alpha$-cyclobutyl alcohol (3ap). A likely explanation was that the highly strained and small cyclobutyl group reduced the steric bulk around the metal center, generating the product in a relatively higher yield. In order to better illustrate the potential application of this reaction for total synthesis and late-stage functionalization, certain substrates containing sensitive functional groups were investigated. Substrates bearing amides and esters were well tolerated (3ax, 3az), while the ones bearing more reactive functional group such as carbonate (3ay) demonstrated a lower yield. Nitriles and nitro-containing substrates were not competible, possibly due to their strong coordinating or oxidation ability.

Additionally, we noticed that the reaction with secondary alcohols both required harsher conditions and produced the corresponding products in relatively lower yields. The result further confirmed the significant steric effect of this reaction. To overcome this challenge, we switched the ligand from PNP L3 to the less bulky L-Et. To our delight, when conducting the reaction under the catalysis of the ruthenium(II)-L-Et complex (Ru-PNP-2), the tested secondary alcohols (3as-3hw) reacted as efficiently as the primary ones, with the exception of 3au due to its very high steric hindrance.

Subsequently, we decided to vary the hydrazones. Parasubstituted benzaldehyde hydrazones were explored first, all of which produced the desired products in moderate to high yields. The $\mathrm{CF}_{3}$ substituted hydrazone demonstrated the lowest yield due to the competing and rapid $\mathrm{WK}$ reduction in the presence of the strong electron-withdrawing effects of $\mathrm{CF}_{3}$ (3ba-3ea). Similarly, most ortho- and meta-substituted benzaldehyde hydrazones proceeded smoothly and produced the desired products in moderate yields. Notably, certain hydrazones with low solubility in the reaction solvent, such as naphthaldehyde hydrazone (3ha), $p$-phenylbenzaldehyde hydrazone (3ba) and p-benzyloxylbenzaldehyde hydrazone (3da), were still able to undergo this transformation smoothly. Aliphatic aldehyde hydrazones proved to be much less reactive (3ks-3ls).

To further evaluate the application potential of this transformation, some naturally occurring complex alcohols, such as $\beta$ Citronellol and (-)-Nopol (5aa-5ab), were examined (Fig. 4). Both of them provided the desired Grignard-type reaction products in good yields. More importantly, the $\pi$-bonds in these natural products were unaffected during the reaction process. The olefin isomerization product as reported in our earlier studies 28 was not observed. A possible reason for the complimentary reactivity could be that in the $\mathrm{PNP}-\mathrm{Ru}$ (II) system, the $\mathrm{H}_{2}$ gas release proceeded much faster than the hydride insertion process. These results demonstrated a great synthetic value for $\mathrm{C}-\mathrm{C}$ bond construction using olefinic natural alcohols, in which the chemoselective Grignard-type reaction of alcohols over olefin transformations could be realized.

Mechanistic studies. A tentative mechanism for this alcoholsurrogated Grignard-type reaction is proposed in Fig. 5 based on previous literature ${ }^{19,27,29,36-41}$ as well as experimental results. The ruthenium(II) catalyst first coordinates with the PNP-pincer ligand L3 to form complex a with the assistance of a base in order to form a highly reactive square planar complex ${ }^{40}$. The alcohol then interacts with complex a to undergo a $\beta$-hydride elimination via a Noyori-type six-membered-ring transition state $\mathbf{b}$ and produces the intermediate $\mathbf{c}^{36}$. This process is also supported by the Density Functional Theory (DFT) calculations (see SI for details). Next, the hydrazone substrate coordinates with the 


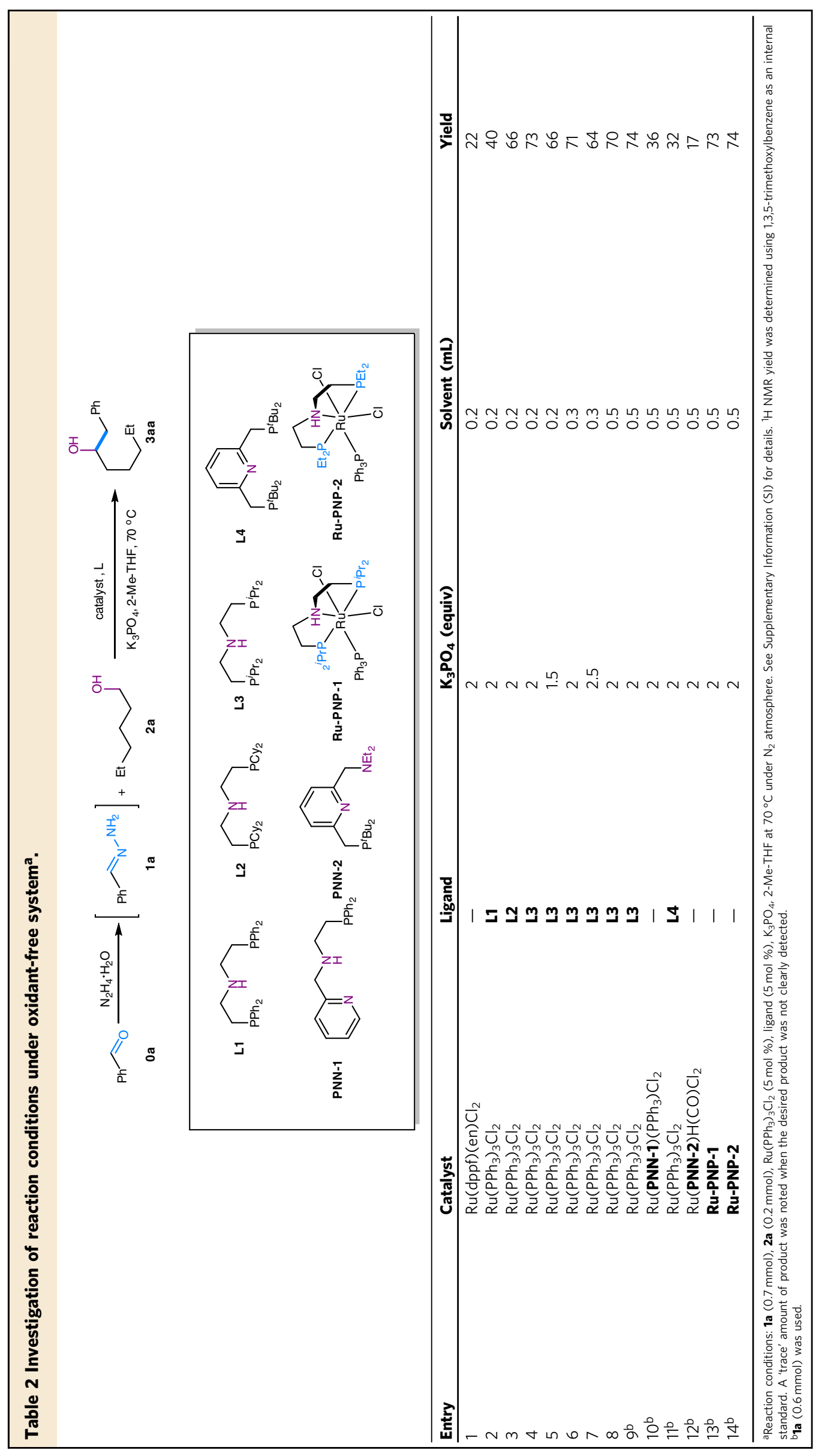




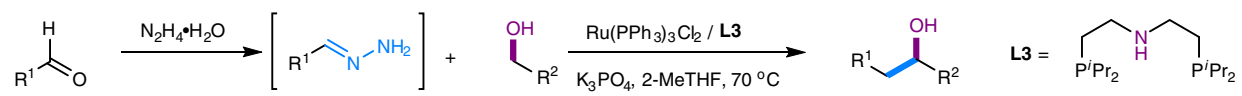
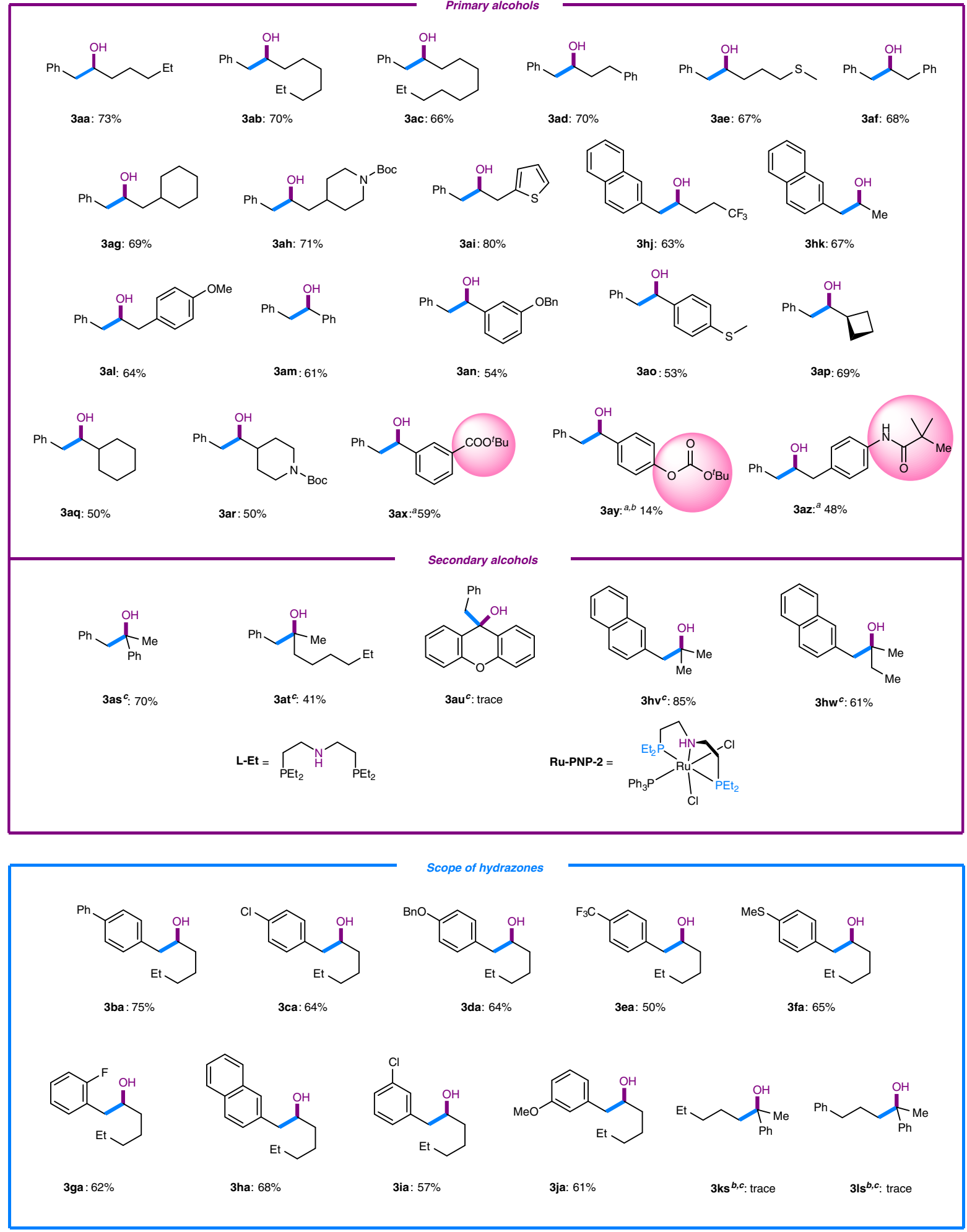

Fig. 3 Substrate scope of the alcohol-surrogated Grignard-type reaction. Reaction conditions: $\mathbf{1 a}(0.6 \mathrm{mmol}), \mathbf{2 a}(0.2 \mathrm{mmol}), \mathrm{Ru}\left(\mathrm{PPh}_{3}\right)_{3} \mathrm{Cl} \mathrm{l}_{2}(5 \mathrm{~mol} \%)$, ligand ( $5 \mathrm{~mol} \%$ ) and $\mathrm{K}_{3} \mathrm{PO}_{4}$ (2 equiv) in 2-MeTHF at $70{ }^{\circ} \mathrm{C}$ under $\mathrm{N}_{2}$ atmosphere for $24 \mathrm{~h}$. See Supplementary Information (SI) for details. Yield of isolated product was reported otherwise noted. a Ru-PNP-1 was used as catalyst. $\mathbf{b}^{1} \mathrm{H}$ NMR yield was determined using mesitylene as an internal standard. c The reaction was conducted at $100{ }^{\circ} \mathrm{C}$, with $\mathbf{1 a}(0.8 \mathrm{mmol})$ and $\mathrm{Ru}-\mathrm{PNP}-2$ being the catalyst. 


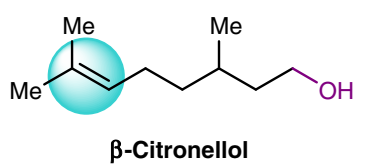

$\beta$-Citronellol

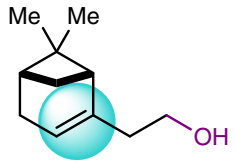

$(-)-\mathrm{Nopol}$<smiles>N/N=C/c1ccccc1</smiles>

$1 \mathrm{a}$
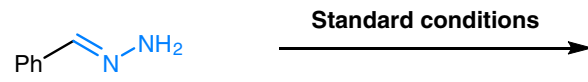

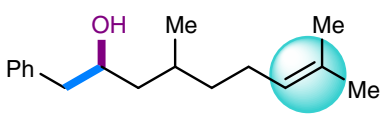

5aa: $67 \%$

No isomerization for olefins

$1 \mathbf{a}$

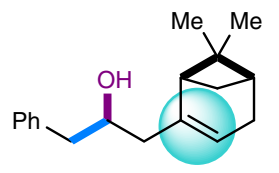

5ab: $62 \%$

Fig. 4 Reactivity of olefinic natural alcohols. Standard reaction conditions: $\left.\mathbf{1 a}(0.6 \mathrm{mmol}), \mathbf{2 a}(0.2 \mathrm{mmol}), \mathrm{Ru}_{(\mathrm{PPh}}\right)_{3} \mathrm{Cl} 2(5 \mathrm{~mol} \%)$, ligand $(5 \mathrm{~mol} \%)$ and $\mathrm{K}_{3} \mathrm{PO}_{4}$ (2 equiv.) in 2-MeTHF at $70^{\circ} \mathrm{C}$ under $\mathrm{N}_{2}$ atmosphere for $24 \mathrm{~h}$. See Supplementary Information (SI) for details. Some naturally available olefinic alcohols also reacted efficiently, in which the olefin did not isomerize with the alcohol being the only reaction site. These two examples demonstrated the synthetic potentials, attributed to the special tolerance to olefins by the ruthenium catalyst.

ruthenium center, which interacts with a hydride and hydrogen gas is released ${ }^{41}$. Concurrently, the 1,2 - addition process via a Zimmerman-Traxler chair-like transition state $\mathbf{d}$ is completed as we proposed previously ${ }^{27}$. Finally, after the $\mathrm{C}-\mathrm{C}$ bond formation and the release of $\mathrm{N}_{2}$ gas, the desired product is formed with the regeneration of the catalyst for the next cycle.

\section{Discussion}

In conclusion, an oxidant-free $\mathrm{Ru}(\mathrm{II})$-PNP catalyzed Grignardtype reaction with alcohol as a carbonyl surrogate was successfully demonstrated. This reaction takes advantage of both the kinetically favored dehydrogenation process provided by a phosphine-amine ligand and the thermodynamic driving force of the 1,2-addition to carbonyls by hydrazone with $\mathrm{Ru}(\mathrm{II})$ catalysis. The development of this transformation marks an evolution in the Grignard-type reaction, wherein direct construction of $\mathrm{C}-\mathrm{C}$ bonds are possible from various naturally abundant alcohols, with a tolerance for sensitive functional groups and further expanding Grignard-type reactions from an early-stage construction to latestage modifications. Future work includes a more in-depth investigation of the application potentials as well as mechanistic studies for this alcohol-surrogated Grignard-type reaction.

\section{Methods}

General procedure for reaction in Table 1. $\mathrm{Ru}\left(\mathrm{PPh}_{3}\right)_{2} \mathrm{Cl}_{2}(0.01 \mathrm{mmol})$, dcypf $(0.01 \mathrm{mmol}), \mathrm{K}_{3} \mathrm{PO}_{4}(0.22 \mathrm{mmol})$ and oxidant $(0.4 \mathrm{mmol}, 2$ equiv)* were added into a V-shaped reaction tube equipped with a stir bar in the glovebox. Then, the reaction tube was sealed and moved out of the glovebox. After that, 1a solution (prepared by the method described in SI, Procedure A, $0.22 \mathrm{~mL}, 0.25 \mathrm{mmol}$ ) was added first, followed by the addition of $\mathbf{2 a}(25.0 \mu \mathrm{L}, 0.2 \mathrm{mmol})$. The mixture was stirred for $24 \mathrm{~h}$. Then, 1,3,5-trimethoxylbenzene $(11.2 \mathrm{mg}, 0.067 \mathrm{mmol})$ was added in the mixture as standard. Then, the solution was filtered by celite and concentrated to dryness. The crude mixture was diluted by $\mathrm{CDCl}_{3}$ to run the ${ }^{1} \mathrm{H}$ NMR test to determine the ${ }^{1} \mathrm{H}$ NMR yield.

*For entry 2, the reaction tube was sealed before exposed to air for $5 \mathrm{~min}$. For entry 3 , after removing the reaction tube out of glovebox, it was charged with $\mathrm{O}_{2}$ via 3 times vacuum-refill by oxygen balloon.

General procedure for reaction in Table 2. $\mathrm{Ru}\left(\mathrm{PPh}_{3}\right)_{2} \mathrm{Cl}_{2}(0.01 \mathrm{mmol})$, ligand $(0.01 \mathrm{mmol})$ and $\mathrm{K}_{3} \mathrm{PO}_{4}(0.4 \mathrm{mmol})$ were added into a $\mathrm{V}$-shaped reaction tube equipped with a stir bar in the glovebox. Then, the reaction tube was sealed and moved out of the glovebox. After that, $0.5 \mathrm{~mL}$ 2-Me-THF was added and followed by the addition of corresponding amount of $\mathbf{1 a}$ (prepared by the method described in SI, Procedure B) and 2a $(25.0 \mu \mathrm{L}, 0.2 \mathrm{mmol})$. The mixture was stirred for $24 \mathrm{~h}$ under $\mathrm{N}_{2}$ at $70^{\circ} \mathrm{C}$. After completion, the solution was filtered by celite and concentrated to dryness. Then, 1,3,5-trimethoxylbenzene (11.2 $\mathrm{mg}, 0.067 \mathrm{mmol})$ was added in the mixture as standard. The crude mixture was diluted by $\mathrm{CDCl}_{3}$ and the ${ }^{1} \mathrm{H}$ NMR test was run to determine the ${ }^{1} \mathrm{H}$ NMR yield.

General procedure for reactions in Figs. 3 and 4. $\mathrm{Ru}\left(\mathrm{PPh}_{3}\right)_{3} \mathrm{Cl}_{2}(0.01 \mathrm{mmol})$, L1 $(0.01 \mathrm{mmol}), \mathrm{K}_{3} \mathrm{PO}_{4}(0.4 \mathrm{mmol})$ and solid substrates were added into a $\mathrm{V}$-shaped reaction tube equipped with a stir bar in the glovebox. Then, the reaction tube was sealed and moved out of the glovebox. After that, $0.5 \mathrm{~mL}$ 2-Me-THF was added first, followed by the addition of liquid substrates. The mixture was stirred under $70^{\circ} \mathrm{C}$ for $24 \mathrm{~h}$. The reaction mixture was filtered through a celite plug and washed with $2-3 \mathrm{~mL} \mathrm{CH}_{2} \mathrm{Cl}_{2}$. The solvent was removed by a rotary evaporator and the residue was purified by column chromatography on silica gel (using hexane and ethyl acetate as eluents) to give the pure product.

General procedure for products 3as-3au, 3hv, and 3hw. Ru-PNP-3 (0.01 $\mathrm{mmol}), \mathrm{K}_{3} \mathrm{PO}_{4}(0.4 \mathrm{mmol})$ and solid substrates were added into a $\mathrm{V}$-shaped reaction tube equipped with a stir bar in the glovebox. Then, the reaction tube was sealed and moved out of the glovebox. After that, $0.5 \mathrm{~mL}$ 2-Me-THF was added first, followed by the addition of liquid substrates. The mixture was stirred under $100^{\circ} \mathrm{C}$ for $24 \mathrm{~h}$. The reaction mixture was filtered through a celite plug and washed with $2-3 \mathrm{~mL} \mathrm{CH}_{2} \mathrm{Cl}_{2}$. The solvent was removed by a rotary evaporator and the residue was purified by column chromatography on silica gel (using hexane and ethyl acetate as eluents) to give the pure product.

General procedure for products 3ax-3az. Ru-PNP-1 (0.01 mmol), $\mathrm{K}_{3} \mathrm{PO}_{4}$ $(0.4 \mathrm{mmol})$ and solid alcohol $(0.2 \mathrm{mmol}$, if applicable) were added into a $\mathrm{V}$-shaped reaction tube equipped with a stir bar in the glovebox. Then, the reaction tube was sealed and moved out of the glovebox. After that, $0.5 \mathrm{~mL} 2$-Me-THF was added first, followed by the addition of $\mathbf{1 a}(0.6 \mathrm{mmol}, 3$ equiv) and liquid alcohols ( $0.2 \mathrm{mmol}$, if applicable). The mixture was stirred under $70^{\circ} \mathrm{C}$ for $24 \mathrm{~h}$. The reaction mixture was filtered through a celite plug and washed with $2-3 \mathrm{~mL} \mathrm{CH} \mathrm{Cl}_{2}$. The solvent was removed by a rotary evaporator and the residue was purified by column chromatography on silica gel (using hexane and ethyl acetate as eluents) to give the pure product. Specifically, for 3ay, the mixture was diluted with $\mathrm{CDCl}_{3}$ and the ${ }^{1} \mathrm{H}$ NMR test was run to determine a trace amount of the desired product.

Procedure for Fig. 3 (3ks, 3/s). $\mathrm{Ru}\left(\mathrm{PPh}_{3}\right)_{3} \mathrm{Cl}_{2}(0.01 \mathrm{mmol}), \mathbf{L 3}(0.006 \mathrm{mmol})$ and $\mathrm{K}_{3} \mathrm{PO}_{4}(0.4 \mathrm{mmol})$ were added into a $\mathrm{V}$-shaped reaction tube equipped with a stir bar in the glovebox. Then, the reaction tube was sealed and moved out of the glovebox. After that, 1 solution (prepared by the method described in Procedure B, $0.55 \mathrm{~mL}, 0.6 \mathrm{mmol}$ ) was added. The mixture was stirred at $100^{\circ} \mathrm{C}$ for $24 \mathrm{~h}$. The reaction mixture was filtered through a celite plug and washed with $2-3 \mathrm{~mL}$ $\mathrm{CH}_{2} \mathrm{Cl}_{2}$. The solvent was removed by a rotary evaporator and mesitylene was added to the residue as internal standard. The mixture was diluted with $\mathrm{CDCl}_{3}$ and the ${ }^{1} \mathrm{H}$ NMR test was run to determine a trace amount of the desired product based on the standard spectrum from literature (see SI for details). 


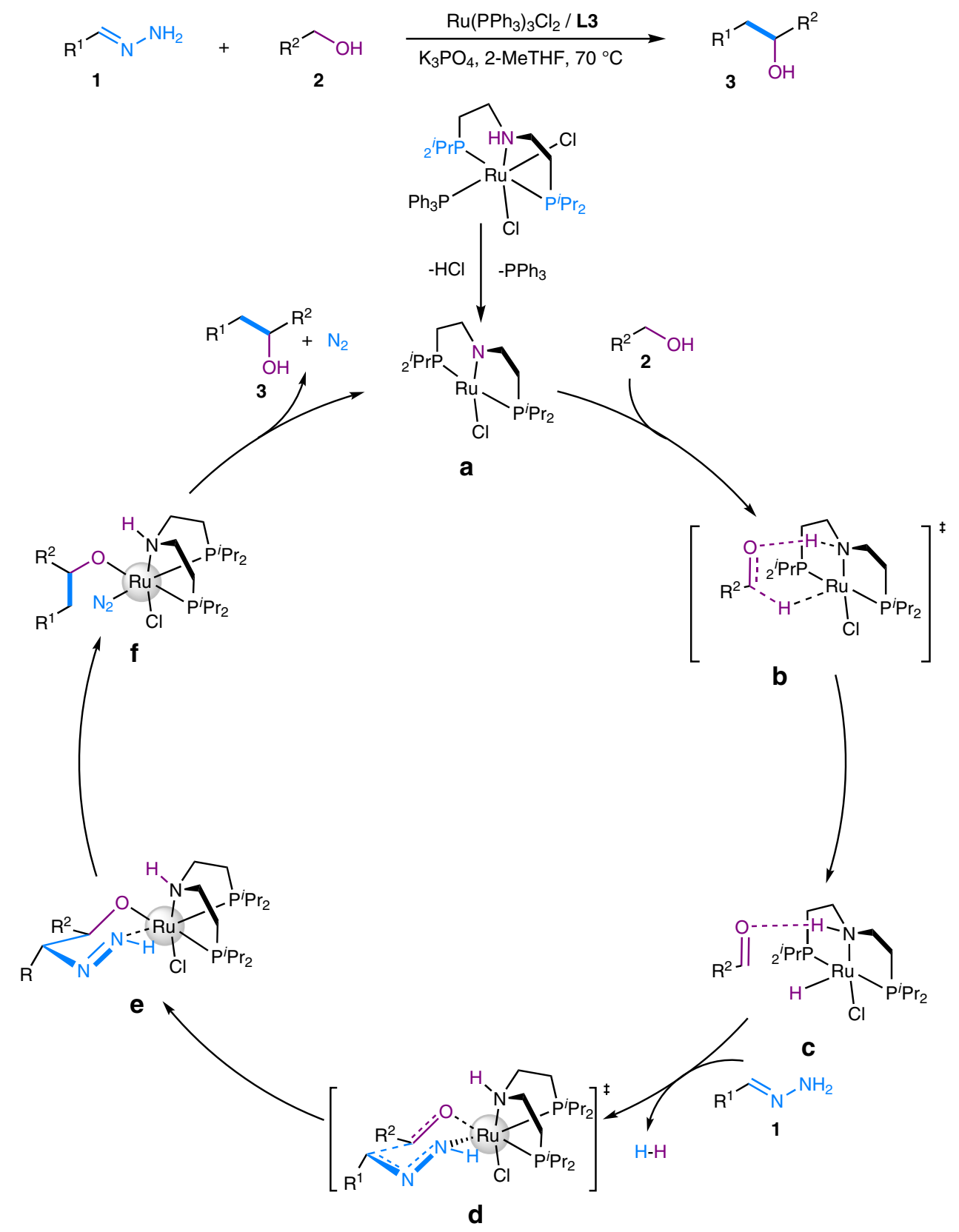

Fig. 5 Proposed mechanism for the alcohol-surrogated Grignard-type reaction. The proposed mechanism starts from the tetracoordinated ruthenium complex. Both the dehydrogenation step and a C-C bond formation step experience six-membered-ring transition states. Specifically, a active species to start the catalytic cycle; $\mathbf{b}$ six-membered-ring transition state for dehydrogenation; $\mathbf{c}$ ruthenium-hydride species after the dehydrogenation step; d transition state for 1,2-addition of hydrazone to carbonyl; e intermediate right after C-C bond formation; $\mathbf{f}$ intermediate after denitrogen which then followed by the formation of desired product.

\section{Data availability}

The authors declare that the data supporting the findings of this study are available within the article and Supplementary Information file, or from the corresponding author upon reasonable request.

Received: 16 April 2020; Accepted: 27 October 2020;

Published online: 26 November 2020

\section{References}

1. Cernak, T., Dykstra, K. D., Tyagarajan, S., Vachal, P. \& Krska, S. W. The medicinal chemist's toolbox for late stage functionalization of drug-like molecules. Chem. Soc. Rev. 45, 546-576 (2016).
2. Grignard, V. Sur quelques nouvelles combinaisons organometalliques du magnésium et leur application à des synthèses d'alcools et d'hydrocarbures. Compt. Rend. 130, 1322 (1900).

3. Shirley, D. A. The synthesis of ketones from acid halides and organometallic compounds of magnesium, zinc, and cadmium. Org. React. 8, 28 (1954).

4. Larock, R. C. Organomercurials in organic synthesis. Tetrahedron 38, 1713-1754 (1982).

5. Knochel, P. \& Jones, P. Organozinc Reagents: a Practical Approach (Oxford University Press, Oxford, 1999).

6. Rappoport, Z. \& Marek, I. The Chemistry of Organolithium Compounds, Volume 1, 2 Volume Set (John Wiley \& Sons, New York, 2004).

7. Smith, M. B. \& March, J. March's Advanced Organic Chemistry: Reactions, Mechanisms, and Structure (John Wiley \& Sons, New York, 2007).

8. Shi, L. et al. A reaction for $\mathrm{sp}^{3}-\mathrm{sp}^{3} \mathrm{C}-\mathrm{C}$ bond formation via cooperation of Lewis acid-promoted/Rh-catalyzed C-H bond activation. J. Am. Chem. Soc. 127, 10836-10837 (2005) 
9. Skucas, E., Zbieg, J. R. \& Krische, M. J. Anti-aminoallylation of aldehydes via ruthenium-catalyzed transfer hydrogenative coupling of sulfonamido allenes: 1,2-aminoalcohols. J. Am. Chem. Soc. 131, 5054-5055 (2009).

10. McInturff, E. L., Yamaguchi, E. \& Krische, M. J. Chiral-anion-dependent inversion of diastereo- and enantioselectivity in carbonyl crotylation via ruthenium-catalyzed butadiene hydrohydroxyalkylation. J. Am. Chem. Soc. 134, 20628-20631 (2012).

11. Spielmann, K., Xiang, M., Schwartz, L. A. \& Krische, M. J. Direct conversion of primary alcohols to 1,2-amino alcohols: enantioselective iridium-catalyzed carbonyl reductive coupling of phthalimido-allene via hydrogen auto-transfer. J. Am. Chem. Soc. 141, 14136-14141 (2019).

12. Bäckvall, J.-E., Chowdhury, R. L. \& Karlsson, U. Ruthenium-catalysed aerobic oxidation of alcohols via multistep electron transfer. J. Chem. Soc. Chem. Commun. 27, 473-475 (1991).

13. Chowdhury, R. L. \& Bäckvall, J.-E. Efficient ruthenium-catalysed transfer hydrogenation of ketones by propan-2-ol. J. Chem. Soc. Chem. Commun. 27, 1063-1064 (1991).

14. Wang, G. Z. \& Bäckvall, J.-E. Ruthenium-catalysed oxidation of alcohols by acetone. J. Chem. Soc. Chem. Commun. 28, 337-339 (1992).

15. Wang, G.-Z., Andreasson, U. \& Bäckvall, J.-E. Aerobic oxidation of secondary alcohols via ruthenium-catalysed hydrogen transfer involving a new triple catalytic system. J. Chem. Soc. Chem. Commun. 30, 1037-1038 (1994).

16. Dijksman, A., W. C. E. Arends, I. \& A. Sheldon, R. Efficient ruthenium-TEMPO-catalysed aerobic oxidation of aliphatic alcohols into aldehydes and ketones. Chem. Commun. 35, 1591-1592 (1999).

17. Edwards, M. G. et al. Borrowing hydrogen: a catalytic route to C-C bond formation from alcohols. Chem. Commun. 40, 90-91 (2004).

18. Nixon, T. D., Whittlesey, M. K. \& Williams, J. M. J. Transition metal catalysed reactions of alcohols using borrowing hydrogen methodology. Dalton Trans. 38, 753-762 (2009).

19. Bertoli, M. et al. Osmium and ruthenium catalysts for dehydrogenation of alcohols. Organometallics 30, 3479-3482 (2011).

20. Obora, Y. Recent advances in a-alkylation reactions using alcohols with hydrogen borrowing methodologies. ACS Catal. 4, 3972-3981 (2014).

21. Kulkarni, N. V., Brennessel, W. W. \& Jones, W. D. Catalytic upgrading of ethanol to n-butanol via manganese-mediated Guerbet reaction. ACS Catal. 8, 997-1002 (2017).

22. Chakraborty, S., Daw, P., Ben David, Y. \& Milstein, D. Manganese-catalyzed a-alkylation of ketones, esters, and amides using alcohols. ACS Catal. 8, 10300-10305 (2018)

23. Yang, F.-L. et al. An efficient homogenized ruthenium(II) pincer complex for N-monoalkylation of amines with alcohols. Eur. J. Org. Chem. 2017, 3481-3486 (2017).

24. Gair, J. J. et al. Reaction of a polyphosphino ruthenium(II) acetate complex with Grignard reagents: halogenation, alkylation and $\beta$-elimination. J. Organomet. Chem. 801, 42-47 (2016).

25. Chen, N., Dai, X.-J., Wang, H. \& Li, C.-J. Umpolung addition of aldehydes to aryl imines. Angew. Chem. Int. Ed. 56, 6260-6263 (2017).

26. Dai, X.-J., Wang, H. \& Li, C.-J. Carbonyls as latent alkyl carbanions for conjugate additions. Angew. Chem. Int. Ed. 56, 6302-6306 (2017).

27. Wang, H., Dai, X.-J. \& Li, C.-J. Aldehydes as alkyl carbanion equivalents for additions to carbonyl compounds. Nat. Chem. 9, 374-378 (2017).

28. Li, C.-J. et al. An old dog with new tricks: enjoin Wolff-Kishner reduction for alcohol deoxygenation and $\mathrm{C}-\mathrm{C}$ bond formations. Synlett 30, 1508-1524 (2019).

29. Li, C.-C. et al. Synergistic relay reactions to achieve redox-neutral a-alkylations of olefinic alcohols with ruthenium(II) catalysis. Angew. Chem. Int. Ed. 59, 4544-4549 (2020).

30. Dai, X.-J. \& Li, C.-J. En route to a practical primary alcohol deoxygenation. J. Am. Chem. Soc. 138, 5433-5440 (2016).

31. Dobereiner, G. E. \& Crabtree, R. H. Dehydrogenation as a substrate-activating strategy in homogeneous transition-metal catalysis. Chem. Rev. 110, 681-703 (2010).

32. Musa, S., Shaposhnikov, I., Cohen, S. \& Gelman, D. Ligand-metal cooperation in PCP Pincer complexes: rational design and catalytic activity in acceptorless dehydrogenation of alcohols. Angew. Chem. Int. Ed. 50, 3533-3537 (2011).

33. Muthaiah, S. \& Hong, S. H. Acceptorless and base-free dehydrogenation of alcohols and amines using ruthenium-hydride complexes. Adv. Synth. Catal. 354, 3045-3053 (2012).

34. Gunanathan, C. \& Milstein, D. Applications of acceptorless dehydrogenation and related transformations in chemical synthesis. Science 341, 1229712 (2013).
35. Chang, W., Gong, X., Wang, S., Xiao, L.-P. \& Song, G. Acceptorless dehydrogenation and dehydrogenative coupling of alcohols catalysed by protic NHC ruthenium complexes. Org. Biomol. Chem. 15, 3466-3471 (2017).

36. Sandoval, C. A., Ohkuma, T., Muñiz, K. \& Noyori, R. Mechanism of asymmetric hydrogenation of ketones catalyzed by BINAP/1,2-diamine -ruthenium(II) complexes. J. Am. Chem. Soc. 125, 13490-13503 (2003).

37. Das, U. K., Chakraborty, S., Diskin-Posner, Y. \& Milstein, D. Direct conversion of alcohols into alkenes by dehydrogenative coupling with hydrazine/hydrazone catalyzed by manganese. Angew. Chem. 130, 13632-13636 (2018).

38. Nielsen, M. et al. Efficient hydrogen production from alcohols under mild reaction conditions. Angew. Chem. Int. Ed. 50, 9593-9597 (2011).

39. Friedrich, A., Drees, M., Käss, M., Herdtweck, E. \& Schneider, S. Ruthenium complexes with cooperative PNP-pincer amine, amido, imine, and enamido ligands: facile ligand backbone functionalization processes. Inorg. Chem. 49, 5482-5494 (2010).

40. Askevold, B., Khusniyarov, M. M., Herdtweck, E., Meyer, K. \& Schneider, S. A square-planar ruthenium(II) complex with a low-spin configuration. Angew. Chem. Int. Ed. 49, 7566-7569 (2010).

41. Garbe, M. et al. Manganese(I)-catalyzed enantioselective hydrogenation of ketones using a defined chiral PNP pincer ligand. Angew. Chem. Int. Ed. 56, 11237-11241 (2017).

\section{Acknowledgements}

We acknowledge the Canada Research Chair Foundation (to C.-J.L.), the CFI, FQRNT Center for Green Chemistry and Catalysis, NSERC, the Killam Research Fellow by the Canadian Council of Arts and McGill University for support of our research. We also thank Y. Kim and S. Luo for proof reading, D. Farajat for language polishing.

\section{Author contributions}

C.-C.L. discovered the reaction. C.-C.L., Z.Q., and Z.-P.C. developed the Ru-PNP catalytic system. C.-C.L. designed and conducted the experiments with the assistance of Z.Q. and Z.-P.C. H.W., M.M.S., and R.Z.K. did or helped doing the DFT calculation. C.-C.L. completed the manuscript with the assistance of Z.Q. and Z.-P.C. C.-J.L. guided the whole project and reviewed the manuscript.

\section{Competing interests}

The authors declare no competing interests.

\section{Additional information}

Supplementary information is available for this paper at https://doi.org/10.1038/s41467 020-19857-9.

Correspondence and requests for materials should be addressed to C.-J.L.

Peer review information Nature Communications thanks Chengjian Zhu and the other, anonymous, reviewer(s) for their contribution to the peer review of this work. Peer reviewer reports are available.

Reprints and permission information is available at http://www.nature.com/reprints

Publisher's note Springer Nature remains neutral with regard to jurisdictional claims in published maps and institutional affiliations.

Open Access This article is licensed under a Creative Commons Attribution 4.0 International License, which permits use, sharing, adaptation, distribution and reproduction in any medium or format, as long as you give appropriate credit to the original author(s) and the source, provide a link to the Creative Commons license, and indicate if changes were made. The images or other third party material in this article are included in the article's Creative Commons license, unless indicated otherwise in a credit line to the material. If material is not included in the article's Creative Commons license and your intended use is not permitted by statutory regulation or exceeds the permitted use, you will need to obtain permission directly from the copyright holder. To view a copy of this license, visit http://creativecommons.org/ licenses/by/4.0/.

(C) The Author(s) 2020 